\title{
LSP1-myosin1e bi-molecular complex regulates focal adhesion dynamics and cell migration
}

Katja Schäringer ${ }^{1 *}$, Sebastian Maxeiner ${ }^{1 *}$, Carmen Schalla ${ }^{1}$, Stephan Rütten ${ }^{2}$, Martin Zenke $^{1}$ and Antonio Sechi ${ }^{1}$

${ }^{1}$ Institute of Biomedical Engineering, Dept. of Cell Biology, RWTH Aachen University, Pauwelsstrasse, 30, D-52074 Aachen, Germany

Electron Microscopy Facility, Institute of Pathology, RWTH Aachen University, Pauwelsstrasse, 30, D-52074 Aachen, Germany

*equal contribution

Corresponding author:

Email: antonio.sechi@rwth-aachen.de

Telephone: +492418085248

Running head: LSP1-myosin1e complex regulates cell migration.

Keywords: Actin cytoskeleton remodelling, focal adhesions, cell motility, macrophages. 


\begin{abstract}
Several cytoskeleton-associated proteins and signalling pathways work in concert to regulate actin cytoskeleton remodelling, cell adhesion and migration. We have recently demonstrated that the bi-molecular complex between the leukocyte-specific protein 1 (LSP1) and myosin1e controls actin cytoskeleton remodelling during phagocytosis. In this study, we show that LSP1 down regulation severely impairs cell migration, lamellipodia formation and focal adhesion dynamics in macrophages. Inhibition of the interaction between LSP1 and myosin1e also impairs these processes resulting in poorly motile cells, which are characterised by few and small lamellipodia. Furthermore, cells in which LSP1-myosin1e interaction is inhibited are typically associated with inefficient focal adhesion turnover. Collectively, our findings show that the LSP1-myosin1e bimolecular complex plays a pivotal role in the regulation of actin cytoskeleton remodelling and focal adhesion dynamics required for cell migration.
\end{abstract}




\section{Introduction}

A large library of actin-associated proteins steer nucleation, cross-linking, capping and elongation of actin filaments. The precise spatial and temporal co-ordination of these functions is fundamental for the movement of cells that is required for many biological events ranging from organ development to tissue repair. The importance of actin cytoskeleton dynamics is emphasised by the onset and progress of diseases due to cells lacking or expressing mutated variants of actin-associated proteins (Mathieson, 2012; Ramaekers and Bosman, 2004). In spite of several studies, the functions of some actin-associated proteins have not been well defined. One of such proteins is the leukocyte-specific protein 1 (LSP1). LSP1 is expressed in several cell types of the immune system such as T-cells, B-cells, macrophages and neutrophils. It is also expressed in myeloid and lymphoid cell lines and, despite its name, in endothelial cells (Jongstra et al., 1994; Jongstra et al., 1988; Jongstra-Bilen et al., 2000; Kadiyala et al., 1990; Liu et al., 2005; Maxeiner et al., 2015; Palker et al., 1998).

The amino-terminal half of LSP1 incorporates $\mathrm{Ca}^{2+}$-binding sites and a coiled-coil region (Jongstra et al., 1988; Klein et al., 1989), suggesting that $\mathrm{Ca}^{2+}$ signalling and dimerization could regulate LSP1 function. The carboxy-terminal half incorporates a caldesmon-like region having a weaker F-actin-binding activity (Zhang et al., 2000; Zhang et al., 2001) and two villin headpiece-like sequences, which primarily mediate the interaction of LSP1 with F-actin (Klein et al., 1990; Wong et al., 2003; Zhang et al., 2001). We have demonstrated that the carboxy-terminal half of LSP1 directly interacts with the $\mathrm{SH} 3$ domain of the molecular motor myosin1e through the noncanonical SH3-binding site AGDMSKKS (Maxeiner et al., 2015). These studies suggest that LSP1 may be involved in the regulation of actin cytoskeleton architecture and dynamics. Indeed, the actin-binding activity of LSP1 is required for the formation of the long, actin-rich cell projections that develop in a wide-ranging variety of cells, which overexpress LSP1 (Howard et al., 1998; Miyoshi et al., 2001; Zhang et al., 2001). 
We have provided a direct evidence that LSP1 regulates actin cytoskeleton dynamics. We found that LSP1 localisation and dynamics at internalisation sites during Fc receptor-mediated phagocytosis, a process that depends on actin dynamics, spatially and temporally overlap with that of the actin cytoskeleton (Maxeiner et al., 2015). Moreover, in LSP1-deficient macrophages and in macrophages in which LSP1myosin1e or LSP1-actin interactions are inhibited, Fcy receptor-mediated phagocytosis is severely reduced (Maxeiner et al., 2015). Given the modulation of actin dynamics by LSP1, it is not surprising that LSP1 has been implicated in the regulation of migration of several cell types including neutrophils, dendritic cells and T-cells (Coates et al., 1991; Howard et al., 1994; Howard et al., 1998; Hwang et al., 2015; Jongstra-Bilen et al., 2000; Koral et al., 2015; Li et al., 2000; Petri et al., 2011).

Although these studies clearly show that LSP1 is involved in the regulation of actin cytoskeleton structural organisation and dynamics, the molecular mechanisms underlying the function of this actin-associated protein are still poorly characterised. Current evidence shows that LSP1 is phosphorylated at serine and threonine sites (Carballo et al., 1996; Huang et al., 1997; Jongstra-Bilen et al., 1990; Matsumoto et al., 1995a; Matsumoto et al., 1993; Wu et al., 2007). In lymphocytes, LSP1 is phosphorylated by protein kinase C (PKC) (Carballo et al., 1996; Matsumoto et al., 1995b; Matsumoto et al., 1993), whereas in neutrophils stimulated with the chemoattractant formyl-methionyl-leucyl-phenylalanine (fMLP), LSP1 is phosphorylated by the mitogen-activated protein (MAP) kinase-activated protein kinase 2 (MK2) (Huang et al., 1997; Wu et al., 2007). Notably, PKC-dependent phosphorylation of LSP1 decreases its localisation with the plasma membrane and the actin cytoskeleton (Matsumoto et al., 1995b; Miyoshi et al., 2001). By contrast, LSP1 phosphorylated by MK2 results in the accumulation of phosphorylated LSP1 at the leading edge of neutrophils (Wu et al., 2007). The importance of the interaction between kinases and LSP1 is further supported by the observation that LSP1 targets 
proteins of the ERK/MAP kinase pathway to the actin cytoskeleton (Harrison et al., 2004). Thus, it is plausible that the localisation of LSP1 to actin-rich structures depends on its phosphorylation status and can be regulated by diverse kinases and signalling pathways.

Regardless the positive or negative regulation of cell migration, it is unquestionable that LSP1 controls this important biological process. By contrast, very little is known about the molecular mechanisms underlying this LSP1 function. For instance, it has been shown that LSP1 participates in a complex with WASP and the Arp2/3 complex (Prasad et al., 2012), two important regulators of actin filament nucleation. Furthermore, LSP1 can also be found in a complex together myosin IIA and one of its regulators, the myosin light chain kinase (Cervero et al., 2018). Since LSP1 does not directly interact with WASP, the Arp2/3 complex and myosin IIA, it is likely that LSP1 is recruited to these complexes via its interaction with F-actin. Notably, we have demonstrated that LSP1 binds to the SH3 domain of myosin1e and that this bimolecular complex is essential for efficient actin cytoskeleton dynamics during Fc $\gamma$ receptor-mediated phagocytosis (Maxeiner et al., 2015).

In this study, we have added another piece to the puzzle describing the modus operandi of LSP1. We have demonstrated that the interaction of LSP1 with myosin $1 e$ is essential for efficient focal adhesion dynamics and zyxin kinetics at these locations. The LSP1-myosin1e binary complex also regulates lamellipodia formation and dynamics. Consequently, interfering with LSP1-myosin1e interaction impaired cell migration. 


\section{Materials and Methods}

\section{Cell culture}

Wild type and genetically modified $\mathrm{J774}$ macrophage cell lines were grown in DMEM supplemented with 10\% fetal calf serum (FCS), $4 \mathrm{mM} \mathrm{L-glutamine,} 100 \mu \mathrm{g} / \mathrm{mL}$ streptomycin, and $100 \mathrm{U} / \mathrm{mL}$ penicillin. The packaging cell line 293T (CRL 11268; ATCC) was grown in DMEM high glucose supplemented with 10\% FCS, 2 mM Lglutamine, $1 \mathrm{mM}$ sodium pyruvate, $100 \mu \mathrm{g} / \mathrm{mL}$ streptomycin, and $100 \mathrm{U} / \mathrm{mL}$ penicillin. All cell lines were grown at $37^{\circ} \mathrm{C}$ and $5 \% \mathrm{CO}_{2}$.

Cloning and generation of genetically modified J774 cells

To generate RFP-zyxin, the coding sequence of zyxin was amplified with the following primer pair: forward 5'-GCTTCGAATTCCATGGCGGCCCCCCGCCCGTCT-3' $\begin{array}{lllll}\text { (containing } & \text { a } & \text { EcoRl }\end{array}$ CTCGAGGATCCTCAGGTCTGGGCTCTAGCAGTGTGGCA-3' (containing a BamHI site) using pMSCV-RFP-Zyxin as the template (Gamper et al., 2016). The amplified product was then cloned into the EcoRI and BamHI site of pWPXL-RFP (Maxeiner et al., 2015). Turquoise-zyxin was cloned as following. The coding sequence of turquoise was amplified from pLL3.7m-mTurquoise2-SLBP (18-126)-IRES-H1mMaroon1 (Addgene vector no. 83842) using the following primer pair: forward 5'CGTTTAAACAGGTATGGTGAGCAAGGGCGA-3' (containing a Pmel site) and reverse 5'-GCAGCGAATTCCCTCCCAGGGAACGCAACATTGAGTA-3' (containing an EcoRI site). The amplified product was then cloned into pWPXL-RFP-Zyxin after excision of RFP using Pmel and EcoRI to generate pWPXL-Turquoise-zyxin. Both RFPzyxin and turquoise-zyxin were sequenced to verify the accuracy of the cloning procedure. To generate cells expressing RFP-zyxin or turquoise-zyxin, wild-type and LSP1-KD J774 cells were transduced with lentiviruses carrying the RFP-zyxin gene, whereas $\mathrm{J774}$ cells expressing the deletion mutant LSP1- $\triangle$ SBS or full-length LSP1 
were transduced with lentiviruses carrying the turquoise-zyxin gene. Generation of lentiviruses and transduction of cells was done as already described (Maxeiner et al., 2015).

Immunofluorescence and live cell imaging

Immunofluorescence labelling was done as previously described (Gamper et al., 2016; Maxeiner et al., 2015; Sechi et al., 2016). For vinculin labelling, cells were fixed with 1\% paraformaldehyde (PFA)/0.5\% Triton X-100 in cytoskeleton buffer for 15 min at RT and then post-fixed with 4\% PFA in cytoskeleton buffer for $10 \mathrm{~min}$ at RT. For EB1 and tubulin labelling, cells were fixed with ice-cold $\left(-20^{\circ} \mathrm{C}\right)$ methanol for $4 \mathrm{~min}$, rehydrated with $0.1 \%$ Triton $\mathrm{X}-100$ in Tris-buffered saline (TBS; $3 x, 5 \mathrm{~min})$, and finally washed with TBS. Vinculin, EB1 and tubulin were detected with the monoclonal antibody hVin1 (Sigma-Aldrich), clone 5 (BD Transduction Laboratories, Heidelberg, Germany) and the rat hybridoma supernatant YL1/2 (Wehland et al., 1983), respectively. The actin cytoskeleton was visualised with Alexa fluorophoreconjugated phalloidin (Life Technologies). For live cell imaging, phase contrast and epifluorescence images were acquired with an Axio Observer Z1 inverted microscope (Carl Zeiss, Jena, Germany) equipped with an EMCCD camera (Evolve Delta, Photometrics, Tucson, AZ) driven by ZEN 2.3 software (Carl Zeiss, Jena, Germany).

Analysis of cell migration and focal adhesion dynamics

J774 cells were plated onto self-made glass-bottomed dishes $(\varnothing 6 \mathrm{~cm}$ ) and their migration was recorded continuously for $24 \mathrm{~h}$ (images were acquired every $5 \mathrm{~min}$ ). The migration of all $\mathrm{J774}$ cell lines was analysed using the Fiji (https://imagej.net/Fiji) plug-in MTrackJ (Meijering et al., 2012) to quantify parameters such as average speed and directionality. Cells that touched neighbouring cells, diving cells and cells that displayed an oscillating movement were excluded from the analysis. Focal adhesion 
dynamics was analysed as previously described (Berginski and Gomez, 2013; Würflinger et al., 2011).

\section{Analysis of lamellipodia dynamics}

Lamellipodia dynamics was visualised by phase contrast microscopy after plating J774 cells at low density onto self-made glass-bottomed dishes $(\varnothing 6 \mathrm{~cm})$. Phase contrast images were acquired every 5 seconds using an EMCCD camera (Cascade 512B, Photometrics, Tucson, AZ, USA) driven by IPLab Spectrum software (Scanalytics, Fairfax, VA, USA). The following parameters were measured: number of cells associated with lamellipodia (\% of total cell number), velocity of lamellipodia spreading and lamellipodia width (measured from the beginning of lamellipodia spreading until the first signs of lamellipodia retraction).

Total internal reflection fluorescence microscopy (TIRF)

TIRF microscopy was performed on an Axio Observer Z1 inverted microscope equipped with a motorized TIRF slider (Zeiss). Excitation of GFP, RFP and Turquoise was done using 488, 561 and $458 \mathrm{~nm}$ laser lines (at 10\% of their nominal output power for 488 and $561,30 \%$ for 458), respectively. The depth of the evanescent field for all wavelengths was $\sim 70 \mathrm{~nm}$. Images were acquired every 10 seconds using an Evolve Delta EMCCD camera driven by ZEN software (Zeiss). For all experiments, exposure time, depth of the evanescent field, and electronic gain were kept constant.

Fluorescence recovery after photobleaching (FRAP)

To analyse focal adhesion kinetics, J774 cells expressing RFP- or Turquoise-tagged zyxin were seeded onto self-made glass-bottomed dishes $(\varnothing 6 \mathrm{~cm})$. For fluorescence recovery after photobleaching, cells were imaged on an Axio Observer Z1 inverted microscope equipped with heating stage and $\mathrm{CO}_{2}$ controller (Zeiss) maintained at a constant temperature of $37^{\circ} \mathrm{C}$. A portion of single focal adhesions (approximately $\varnothing$ 
$3.84 \mu \mathrm{m}$ ) was photobleached using a $405 \mathrm{~nm}$ laser driven by the UGA-40 control unit (Rapp Opto Electronic GmbH, Wedel, Germany). The recovery of the fluorescent signal was monitored by imaging cells every second for $15 \mathrm{~min}$. Imaging was done using an Evolve Delta EMCCD camera driven by ZEN software (Zeiss). For all experiments the size of the bleached area, and the duration and intensity of the laser impulse were kept constant. The extent of recovery of the fluorescent signal was determined using Fiji to measure the average pixel intensity values within three distinct regions of interest (ROIs): ROI1: bleached area; ROI2: unbleached area within the cell; and ROI3: background. Normalised FRAP recovery curves and the mobile fractions were calculated using the program easyFRAP (Rapsomaniki et al., 2012).

\section{Scanning and transmission electron microscopy}

For scanning electron microscopy, cells were fixed and processed as already described (Maxeiner et al., 2015; Sechi et al., 2016). Samples were examined with a digital scanning electron microscope (ESEM XL30 FEG; FEI, Hillsboro, OR) using a working distance of $8 \mathrm{~mm}$ and an acceleration voltage of $10 \mathrm{kV}$.

\section{Statistical analysis}

Graphs and statistical tests were done using Prism 8 (GraphPad Software, La Jolla, CA). Differences between sample pairs were analysed using the two-tailed MannWhitney nonparametric $U$ test. The null hypotheses (the two samples have the same median values, that is, they are not different) were rejected when $p>0.5$. For the box-and-whiskers plots, the line in the middle of the box indicates the median, the top of the box indicates the 75th quartile, and the bottom of the box indicates the 25th quartile. Whiskers represent the 10th (lower) and 90th (upper) percentiles. 


\section{Results}

LSP1 is essential for efficient migration of J774 mouse macrophages

Since LSP1 directly interacts with F-actin (Wong et al., 2003) and regulates the dynamics of the actin cytoskeleton (Maxeiner et al., 2015), we hypothesised that LSP1 could control cell migration. To this end, we focused on macrophages because LSP1 is essential for another actin-dependent process in this cell type, namely Fcy receptormediated phagocytosis (Maxeiner et al., 2015). After seeding control cells or cells in which LSP1 was down regulated by shRNA (Maxeiner et al., 2015), we imaged cell migration by phase contrast microscopy over a period of 24 hours. Typically, control cells were characterised by a polarised morphology and the formation of large lamellipodia, which developed in the direction of movement (Fig. 1A and Fig. 1SUP). Furthermore, control $\mathbf{J 7 7 4}$ cells usually travelled large distances (Fig. 1A, C). Conversely, LSP1-deficient $\mathrm{J774}$ cells rarely formed lamellipodia and moved over short distances (Fig. 1B, D and Fig. 1SUP). Consistent with these observations, the average speed of LSP1-deficent $\mathrm{J774}$ cells was significantly smaller than that of control cells $(0.01083 \mu \mathrm{m} / \mathrm{sec}$ for LSP1-deficient cells $(n=138)$ vs. $0.02889 \mu \mathrm{m} / \mathrm{sec}$ for control cells (Fig. 1E; $n=149$ ). These findings clearly show that LSP1 is essential for efficient migration of $\mathrm{J} 774$ macrophages.

LSP1 is essential for normal development of microfilaments, microtubules and focal adhesions

The morphological features and largely decreased migration of LSP1-deficient cells suggest that LSP1 is involved in the organisation of actin and microtubule cytoskeletons as well as cell-substrate adhesion (i.e., focal adhesions). We verified this hypothesis by labelling control and LSP1-deficient J774 cells with anti-tubulin and anti-EB1 antibodies (for assessing microtubule organisation) or fluorescent phalloidin and anti-vinculin antibodies (for assessing microfilaments and focal adhesions, 
respectively). Using TIRF microscopy, we found that control cells developed a prominent microtubule network characterised by long microtubules emanating from a perinuclear area and projecting toward the cell periphery (Fig. 2A, arrows in inset). As expected, peripheral microtubule ends were labelled with EB1, a plus-end protein that regulates microtubule dynamics (Fig. 2A, arrows in inset). By contrast, the microtubule network in LSP1-deficient cells, which were round and smaller than control cells, was formed by short microtubules (Fig. 2B, arrows in inset). In these cells, we could not find any gross alteration of EB1 distribution (Fig. 2B, arrows in inset). Next, we analysed control and LSP1-deficient J774 cells labelled with Alexa 594-phalloidin and anti-vinculin antibody to visualise actin cytoskeleton and focal adhesion by TIRF microscopy, respectively. Control cells were characterised by a spread and elongated morphology with one or multiple large actin-rich lamellipodia at their periphery (Fig. 2C, green arrowheads). These cells interacted with the substratum via several elongated focal adhesions (Fig. 2C, arrows). At variance with these morphological features, LSP1-deficent cells were smaller and round with no or a single small actin-rich lamellipodium (Fig. 2D, red arrowhead). Focal adhesions in these cells were strongly reduced in size and number and showed a rounded shape (Fig. 2D, arrows). Overall, these findings demonstrate that LSP1 is essential for the normal development of microfilaments, microtubules and focal adhesions. They also suggest that LSP1-dependent regulation of cell migration is exerted via the control of these cytoskeletal structures.

LSP1 is essential for the regulation of focal adhesion dynamics

Focal adhesions are highly dynamic structures whose spatial and temporal regulation is essential for cell migration (Sechi and Wehland, 2004; Zamir and Geiger, 2001). The impaired cell migration and formation of focal adhesions in LSP1-deficent J774 cells suggests that LSP1 plays an important role in the control of focal adhesion dynamics. To test this assumption, we engineered control and LSP1-deficient J774 
cells to express RFP-tagged zyxin, a focal adhesion component, to visualise focal adhesion dynamics using TIRF microscopy (Gamper et al., 2016; Sechi et al., 2016) and analysed their dynamics using a dedicated algorithm (Würflinger et al., 2011). The initial examination of time-lapse sequences revealed that focal adhesions in control cells were highly dynamic assembling or disassembling within short time periods (arrows in Fig. 3A and corresponding video). On the contrary, focal adhesions in LSP1-deficient cells appeared to be less dynamic requiring longer time periods to assemble and disassemble (arrows in Fig. 3B and corresponding video). The quantification of several focal adhesion parameters confirmed the impression provided by visually inspecting time-lapse sequences. Precisely, for both growing or shrinking focal adhesions, the change of the area over time, a proxy for assembling and disassembly rates, was faster for focal adhesions in control cells than in LSP1deficient cells (Fig. 3C, D). Accordingly, the assembly and disassembly rates of focal adhesions in LSP1-deficient cells were significantly lower than the corresponding parameters for control focal adhesions (Fig. 3E). Furthermore, the average area was significantly reduced in focal adhesions in LSP1-deficient cells (Fig. 3F), whereas we could not see any difference in their shape (elongation index, Fig. 3G). Finally, the focal adhesion movement relative to the substratum (focal adhesion speed) was also significantly impaired in LSP1-deficient cells (Fig. 3H). These findings clearly show that LSP1 regulates cell migration via the modulation of focal adhesion formation and dynamics.

LSP1-myosin 1e binary complex is essential for the regulation of cell migration We have demonstrated that LSP1 directly interacts with myosin 1e through a noncanonical SH3-binding site. Moreover, downregulating LSP1 or blocking its interaction with myosin1e results in severely impaired Fcy receptor-mediated phagocytosis and the inhibition of actin accumulation and lamellipodia formation around the particles to be internalised (Maxeiner et al., 2015). Since LSP1 deficiency 
impairs cell migration, we reasoned that the LSP1-myosin1e binary complex could play an important role in the regulation of $\mathbf{J 7 7 4}$ cell migration. To experimentally verify this hypothesis, we scrutinised the migration of $\mathrm{J774}$ cells expressing the deletion mutant LSP1- $\triangle$ SBS, which cannot bind to myosin1e (Maxeiner et al., 2015), by phase contrast microscopy for 24 hours. As control, we used J774 cells reexpressing full-length LSP1 (rescue). As expected, cells in which LSP1 was reexpressed did not shown any sign of migration defect highly resembling control J774 cells (Fig. 4B, C, E). By contrast, it was immediately evident that cells expressing LSP1- $\triangle$ SBS travelled very short distances (Fig. 4A, D) and moved at a significantly reduced speed (Fig. 4E). Remarkably, the motile phenotype and the speed of cells expressing LSP1- $\triangle$ SBS was undistinguishable from LSP1-deficient cells (compare with Fig. 1). These observations clearly demonstrate that the binary complex between LSP1 and myosin1e is essential for efficient J774 migration.

LSP1-myosin 1e binary complex is necessary for lamellipodia activity

One of the earliest events of cell migration is the formation and stabilisation of one lamellipodium in the direction of movement. Because cell migration is severely impaired in LSP1-deficient cells and in cells in which LSP1 cannot interact with myosin1e, we decided to determine whether lamellipodia activity is compromised in these cell types. The closer inspection of time-lapse sequences at high magnification revealed that motile control cells frequently form one large and persistent lamellipodium in the direction of movement (Fig. 5A and corresponding video). LSP1-deficient cells and cells expressing LSP1- $\triangle$ SBS greatly differed from this phenotype in that they maintained a round morphology and never formed a large and persistent lamellipodium (Fig. 5B, C). These cells were rather characterised by the formation of small lamellipodia that formed around their periphery (Fig. 5B, C and corresponding video). Consistent with this visual examination, we found that the frequency of lamellipodia formation and its width were significantly lower in LSP1- 
deficient cells and cells expressing LSP1- $\triangle$ SBS (Fig. 5D, F). Interestingly, the speed of lamellipodia spreading was significantly reduced in LSP1- $\triangle$ SBS cells, but not in LSP1-deficient cells (Fig. 5E) possibly due to a large data variability. Collectively, these findings demonstrate that LSP1 and myosin1e are indispensable for efficient lamellipodia activity.

LSP1-myosin 1e interaction regulates the dynamics and kinetics of LSP1

Next, we determined whether the interaction with myosin1e affected the dynamics and kinetics of LSP1. We initially visualised LSP1 localisation and dynamics by TIRF microscopy over a period of 10-15 minutes. In control cells and cells re-expressing full-length LSP1 (rescue), LSP1 was highly dynamic often localising to lamellipodia (asterisk in Fig. 6A and C; see also corresponding video) and to filamentous-like and focal adhesions-like structures (arrows in Fig. 6A and C). In cells expressing LSP1$\triangle$ SBS, LSP1 appeared to be less dynamics and was concentrated at the perinuclear area and at very small lamellipodia (arrows in Fig. 6B; see also corresponding video). To corroborate the visual impression that LSP1 was less dynamic when unable to interact with myosin 1e, we determined its kinetics using FRAP microscopy. As shown in Fig. 6D and $E$, both the time-course of the fluorescence recovery and the mobile fraction of LSP1 in control cells and cells re-expressing full-length LSP1 (rescue) were indistinguishable. Conversely, the time-course of the fluorescence recovery and the mobile fraction of LSP1- $\triangle$ SBS was significantly reduced (Fig. 6, D, E). Thus, our findings suggest that efficient LSP1 dynamics and kinetics depend on its interaction with myosin1e.

LSP1-myosin1e binary complex is essential for the regulation of focal adhesion dynamics

The above findings provide clear evidence that LSP1 deficiency severely impairs cell migration, focal adhesion dynamics and lamellipodia activity. Moreover, the 
interaction of LSP1 with myosin1e is essential for the regulation of cell migration and lamellipodia formation. Based on these observations and given the importance of focal adhesion dynamics for cell migration, we sought to determine whether LSP1myosin1e binary complex also plays a role in the regulation of focal adhesion dynamics. For this purpose, we genetically modified J774 expressing LSP1- $\triangle$ SBS or full-length LSP1 (rescue) with turquoise-zyxin to visualised focal adhesion dynamics using TIRF microscopy.

Similar to LSP1-deficient cells, LSP1- $\triangle$ SBS cells were characterised by few and small focal adhesions with a slower turnover (Fig. 7B and corresponding video). As expected, focal adhesions in cells re-expressing full-length LSP1 were similar to those in control cells (Fig. 7A and corresponding video; compare with Fig. 3A). Detailed analysis of focal adhesion dynamics using a dedicated algorithm supported this visual impression showing that assembly and disassembly rates as well as size were significantly reduced in LSP1- $\triangle$ SBS cells (Fig. 7C-H). Consistent with these observations, we found a reduced amount of vinculin in the actin cytoskeleton fraction of LSP1-deficient and LSP1- $\triangle$ SBS cells (Fig. 8).

Next, as a complementary approach to demonstrate the role of LSP1-myosin 1e binary complex in the regulation of focal adhesions, we analysed zyxin kinetics by fluorescence recovery after photobleaching. To this end, focal adhesions in control, LSP1-deficient, LSP1- $\triangle$ SBS and cells re-expressing full-length LSP1 (rescue) were bleached and the recovery of zyxin fluorescence within the bleached areas was monitored over a period of several minutes. According to the above analysis of focal adhesion dynamics, zyxin kinetics was not significantly different in control cells and cells re-expressing full-length LSP1 (Fig. 9A, D-F). Conversely, zyxin recovery was much slower and less complete at focal adhesions in LSP1-deficient and LSP1- $\triangle$ SBS cells (Fig. 9B, C, E, F). Taken together, these findings demonstrate that the LSP1myosin1e binary complex is required for the regulation of focal adhesion dynamics. 


\section{Discussion}

Deciphering the mechanisms of cell migration is essential for fully understanding this process in normal and pathological conditions. In this study, we demonstrate that LSP1 is essential for efficient cell migration in $\mathrm{J774}$ macrophages. LSP1 controls this process through the regulation of the formation and dynamics of lamellipodia and focal adhesions. In this context, the interaction of LSP1 with the molecular motor myosin1e is indispensable for LSP1 function in cell migration since inhibiting the formation of LSP1-myosin1e binary complex severely impairs cell migration, formation and dynamics of lamellipodia and focal adhesions. These findings provide novel insights into the regulation of cell migration in immune cells and demonstrate a pivotal role for the LSP1-myosin1e binary complex in the regulation of this process.

Previous investigations have addressed the role of LSP1 in cell migration in a variety of cell types. For instance, LSP1 deficiency significantly reduces the migration of dendritic cells induced by gp120 (Anand et al., 2009; Prasad et al., 2012). Likewise, LSP1\% neutrophils show both impaired migration and chemotactic response (Hannigan et al., 2001). Using a complementary approach, it was shown that the expression of LSP1 to physiological levels in a myeloid cell line, which does not express LSP1, results in a significant enhancement of cell migration (Li et al., 2000). Notably, large lamellipodia are rarely observed in LSP1 ${ }^{-/-}$cells, which rather form small and transient lamellipodia (Hannigan et al., 2001). In this study, we have deepened our knowledge of LSP1 function demonstrating that its deficiency severely impairs the formation of lamellipodia, focal adhesion dynamics and cell migration in macrophages. Hence, LSP1 unequivocally plays a key role in the regulation of cell migration in different cellular systems.

For the sake of clarity, it should be mentioned that other studies show that the loss, or reduced expression, of LSP1 increases rather than reduces cell migration. 
However, this effect has been described in pathological situations such as in T-cells derived from patients with rheumatoid arthritis and in hepatoma cells (Hwang et al., 2015; Koral et al., 2015). Since, in hepatoma cells, a classical scratch assay and not single-cell tracking was used to study cell migration, it is not possible to rule out that the described increase of cell migration was not due to the increase of cell proliferation observed in LSP1/- cells. In addition, the marginal (1.36-fold) increase of migration of LSP1\% neutrophils could be observed when cells were seeded only on fibrinogen, but not on other substrates (Wang et al., 2002). Hence, when interpreting these studies ascribing a negative regulatory effect on cell migration to LSP1, experimental parameters that may impact on this effect must be considered in order to avoid inaccurate interpretations.

Although it is known that LSP1 interacts with actin filaments (Klein et al., 1990; Wong et al., 2003; Zhang et al., 2001), the mechanisms underlying LSP1 function in the regulation of actin-driven processes are, as yet, poorly defined. Before discussing potential scenarios describing LSP1 function, our previous study (Maxeiner et al., 2015) and the findings described here clearly indicate that one possible mechanism for LSP1 function requires its interaction with myosin1e. In this context, we envisage that LSP1 and myosin1e will synergise to activate signalling pathways specific for actin cytoskeleton remodelling. How does, then, this binary complex regulate cell migration and focal adhesion dynamics? It has been shown that LSP1 is a component of a complex including WASP and the Arp2/3 complex (Prasad et al., 2012). LSP1 also co-precipitates with regulators of myosin II such as calmodulin and the myosin light chain kinase (Cervero et al., 2018). Since WASP and the Arp2/3 complex are essential for actin filament nucleation in lamellipodia and myosin II activity controls actin cytoskeleton contraction and cell body displacement required for cell migration (Blanchoin et al., 2014), it is reasonable to envisage a signalling scenario in which LSP1 controls both actin filament nucleation and cell body translocation (Fig. 10). Our 
present study supports this hypothesis since in LSP1-deficient cells both lamellipodia formation and cell migration are severely impaired.

It is interesting to note that targeting myosin1e to mitochondria induces the accumulation at the surface of these organelles of actin filaments and activators of the Arp2/3 complex WIP and N-WASP (Cheng et al., 2012). Furthermore, in yeast, a type I myosin has been involved in actin filament formation induced by WASP and the Arp2/3 complex (Sirotkin et al., 2005). These studies suggest a role for myosin1e in the formation of actin filaments. This role for myosin1e is further supported by two additional studies showing that lamellipodia formation and stability is impaired in myosin1e-deficient cells (Gupta et al., 2013; Tanimura et al., 2016). It is also important to note that cells expressing a variant of myosin1e lacking the $\mathrm{SH} 3$ domain fail to form mature focal adhesions (Gupta et al., 2013). Consistent with these studies, we have demonstrated that lamellipodia and focal adhesion formation are impaired in LSP1-deficent cells and in cells expressing a variant of LSP1 that cannot interact with myosin 1e. Thus, it is conceivable that LSP1 and myosin1e work in concert to regulate actin filament assembly through WASP-family proteins and the Arp $2 / 3$ complex (Fig. 10). In this context, it should also be taken into account that myosin 1e has been found in actin-rich structures containing FHOD1 (Gupta et al., 2013). In mammalian cells, FHOD1 stimulates the formation of stress fibres and cell migration and is recruited to integrin clusters (Iskratsch et al., 2013; Koka et al., 2003; Schulze et al., 2014). Moreover, FHOD1 knockdown impairs cell spreading and focal adhesion maturation (Iskratsch et al., 2013). In Drosophila, the mutation of the FHOD1 homologue Knittrig results in smaller macrophages, which show reduced migration (Lammel et al., 2014). These observations are, again, in agreement with our findings showing a similar phenotype in LSP1-deficent cells and in cells expressing a variant of LSP1 that cannot interact with myosin1e. Hence, we propose that the LSP1-myosin1e binary complex exert its function also via FHOD1 (Fig. 10). 
Finally, it is important to note that the prominence of LSP1 in the regulation of cell migration is further supported by studies on pathogen-cell interactions. It has been clearly demonstrated that several types of pathogens such as Listeria monocytogenes and vaccinia viruses have developed elegant strategies to subvert fundamental steps of actin cytoskeleton remodelling for their spreading and survival (Geese et al., 2002; May et al., 1999; Pust et al., 2005; Way, 1998). In this context, it has been shown that the human immunodeficiency virus can induce migration of dendritic cells (Anand et al., 2009). These viruses control dendritic cell migration by the binding of their envelope protein gp120 to DC-SIGN on dendritic cells (Prasad et al., 2017; Prasad et al., 2012). Remarkably, gp120-DC-SIGN interaction triggers a signalling cascade that involves LSP1 and leads to Rho GTPase (a regulator of focal adhesion dynamics) activation (Anand et al., 2009; Prasad et al., 2017; Prasad et al., 2012). This study, thus, support our findings highlighting the key role of LSP1 in the regulation of focal adhesion dynamics and cell migration.

Collectively, our findings provide novel evidence about LSP1 function and its ability to regulate two crucial aspects of cell migration in co-operation with myosin1e: a) actin cytoskeleton assembly (required for lamellipodia formation and dynamics) and b) focal adhesion formation and dynamics. Several questions remain to be addressed. For instance, is the function of myosin1e dependent on its interaction with LSP1? Is LSP1 phosphorylation required for its role in cell migration? Has LSP1 other binding partners in addition to myosin1e? Could LSP1 be a target for novel pharmaceutical treatments of HIV infections? The answers to these questions will certainly help to better understand the role of LPS1 and its interaction with myosin1e not only in the regulation of cell migration and adhesion but also in other processes dependent on actin cytoskeleton remodelling. 
bioRxiv preprint doi: https://doi.org/10.1101/2020.02.26.963991; this version posted February 27, 2020. The copyright holder for this

preprint (which was not certified by peer review) is the author/funder. All rights reserved. No reuse allowed without permission.

\section{Acknowledgements}

We thank Ms. Gülcan Aydin for excellent technical assistance. 


\section{References}

Anand, A.R., A. Prasad, R.R. Bradley, Y.S. Deol, T. Nagaraja, X. Ren, E.F. Terwilliger, and R.K. Ganju. 2009. HIV-1 gp120-induced migration of dendritic cells is regulated by a novel kinase cascade involving Pyk2, p38 MAP kinase, and LSP1. Blood. 114:3588-3600.

Berginski, M.E., and S.M. Gomez. 2013. The Focal Adhesion Analysis Server: a web tool for analyzing focal adhesion dynamics. F1000Res. 2:68.

Blanchoin, L., R. Boujemaa-Paterski, C. Sykes, and J. Plastino. 2014. Actin dynamics, architecture, and mechanics in cell motility. Physiol Rev. 94:235-263.

Carballo, E., D. Colomer, J.L. Vives-Corrons, P.J. Blackshear, and J. Gil. 1996. Characterization and purification of a protein kinase $C$ substrate in human $B$ cells. Identification as lymphocyte-specific protein 1 (LSP1). Journal of immunology. 156:1709-1713.

Cervero, P., C. Wiesner, A. Bouissou, R. Poincloux, and S. Linder. 2018. Lymphocytespecific protein 1 regulates mechanosensory oscillation of podosomes and actin isoform-based actomyosin symmetry breaking. Nat Commun. 9:515.

Cheng, J., A. Grassart, and D.G. Drubin. 2012. Myosin 1E coordinates actin assembly and cargo trafficking during clathrin-mediated endocytosis. Molecular biology of the cell. 23:2891-2904.

Coates, T.D., J.C. Torkildson, M. Torres, J.A. Church, and T.H. Howard. 1991. An inherited defect of neutrophil motility and microfilamentous cytoskeleton associated with abnormalities in 47-Kd and 89-Kd proteins. Blood. 78:13381346.

Gamper, I., D. Fleck, M. Barlin, M. Spehr, S. El Sayad, H. Kleine, S. Maxeiner, C. Schalla, G. Aydin, M. Hoss, D.W. Litchfield, B. Luscher, M. Zenke, and A. Sechi. 2016. GAR22beta regulates cell migration, sperm motility, and axoneme structure. Molecular biology of the cell. 27:277-294.

Geese, M., J.J. Loureiro, J.E. Bear, J. Wehland, F.B. Gertler, and A.S. Sechi. 2002. Contribution of Ena/VASP proteins to intracellular motility of listeria requires phosphorylation and proline-rich core but not F-actin binding or multimerization. Molecular biology of the cell. 13:2383--2396.

Gupta, P., N.C. Gauthier, Y. Cheng-Han, Y. Zuanning, B. Pontes, M. Ohmstede, R. Martin, H.J. Knolker, H.G. Dobereiner, M. Krendel, and M. Sheetz. 2013. Myosin 1E localizes to actin polymerization sites in lamellipodia, affecting actin dynamics and adhesion formation. Biol Open. 2:1288-1299.

Hannigan, M., L. Zhan, Y. Ai, and C.K. Huang. 2001. Leukocyte-specific gene 1 protein (LSP1) is involved in chemokine KC-activated cytoskeletal reorganization in murine neutrophils in vitro. Journal of leukocyte biology. 69:497-504.

Harrison, R.E., B.A. Sikorski, and J. Jongstra. 2004. Leukocyte-specific protein 1 targets the ERK/MAP kinase scaffold protein KSR and MEK1 and ERK2 to the actin cytoskeleton. Journal of cell science. 117:2151-2157. 
Howard, T., Y. Li, M. Torres, A. Guerrero, and T. Coates. 1994. The 47-kD protein increased in neutrophil actin dysfunction with 47- and 89-kD protein abnormalities is lymphocyte-specific protein. Blood. 83:231-241.

Howard, T.H., J. Hartwig, and C. Cunningham. 1998. Lymphocyte-specific protein 1 expression in eukaryotic cells reproduces the morphologic and motile abnormality of NAD 47/89 neutrophils. Blood. 91:4786-4795.

Huang, C.K., L. Zhan, Y. Ai, and J. Jongstra. 1997. LSP1 is the major substrate for mitogen-activated protein kinase-activated protein kinase 2 in human neutrophils. The Journal of biological chemistry. 272:17-19.

Hwang, S.H., S.H. Jung, S. Lee, S. Choi, S.A. Yoo, J.H. Park, D. Hwang, S.C. Shim, L. Sabbagh, K.J. Kim, S.H. Park, C.S. Cho, B.S. Kim, L. Leng, R.R. Montgomery, R. Bucala, Y.J. Chung, and W.U. Kim. 2015. Leukocyte-specific protein 1 regulates T-cell migration in rheumatoid arthritis. Proc Natl Acad Sci U S A. 112:E6535-6543.

Iskratsch, T., C.H. Yu, A. Mathur, S. Liu, V. Stevenin, J. Dwyer, J. Hone, E. Ehler, and M. Sheetz. 2013. FHOD1 is needed for directed forces and adhesion maturation during cell spreading and migration. Dev Cell. 27:545-559.

Jongstra, J., M.E. Ittel, N.N. Iscove, and G. Brady. 1994. The LSP1 gene is expressed in cultured normal and transformed mouse macrophages. Molecular immunology. 31:1125-1131.

Jongstra, J., J. Jongstra-Bilen, G.F. Tidmarsh, and M.M. Davis. 1988. The in vitro translation product of the murine lambda 5 gene contains a functional signal peptide. Molecular immunology. 25:687-693.

Jongstra-Bilen, J., V.L. Misener, C. Wang, H. Ginzberg, A. Auerbach, A.L. Joyner, G.P. Downey, and J. Jongstra. 2000. LSP1 modulates leukocyte populations in resting and inflamed peritoneum. Blood. 96:1827-1835.

Jongstra-Bilen, J., A.J. Young, R. Chong, and J. Jongstra. 1990. Human and mouse LSP1 genes code for highly conserved phosphoproteins. Journal of immunology. 144:1104-1110.

Kadiyala, R.K., B.W. Mclntyre, and A.M. Krensky. 1990. Molecular cloning and characterization of WP34, a phosphorylated human lymphocyte differentiation and activation antigen. European journal of immunology. 20:2417-2423.

Klein, D.P., S. Galea, and J. Jongstra. 1990. The lymphocyte-specific protein LSP1 is associated with the cytoskeleton and co-caps with membrane IgM. Journal of immunology. 145:2967-2973.

Klein, D.P., J. Jongstra-Bilen, K. Ogryzlo, R. Chong, and J. Jongstra. 1989. Lymphocyte-specific Ca2+-binding protein LSP1 is associated with the cytoplasmic face of the plasma membrane. Molecular and cellular biology. 9:3043-3048.

Koka, S., C.L. Neudauer, X. Li, R.E. Lewis, J.B. McCarthy, and J.J. Westendorf. 2003. The formin-homology-domain-containing protein FHOD1 enhances cell migration. Journal of cell science. 116:1745-1755. 
Koral, K., S. Paranjpe, W.C. Bowen, W. Mars, J. Luo, and G.K. Michalopoulos. 2015. Leukocyte-specific protein 1: a novel regulator of hepatocellular proliferation and migration deleted in human hepatocellular carcinoma. Hepatology. 61:537-547.

Lammel, U., M. Bechtold, B. Risse, D. Berh, A. Fleige, I. Bunse, X. Jiang, C. Klambt, and S. Bogdan. 2014. The Drosophila FHOD1-like formin Knittrig acts through Rok to promote stress fiber formation and directed macrophage migration during the cellular immune response. Development. 141:1366-1380.

Li, Y., Q. Zhang, R. Aaron, L. Hilliard, and T.H. Howard. 2000. LSP1 modulates the locomotion of monocyte-differentiated U937 cells. Blood. 96:1100-1105.

Liu, L., D.C. Cara, J. Kaur, E. Raharjo, S.C. Mullaly, J. Jongstra-Bilen, J. Jongstra, and P. Kubes. 2005. LSP1 is an endothelial gatekeeper of leukocyte transendothelial migration. The Journal of experimental medicine. 201:409418.

Mathieson, P.W. 2012. The podocyte cytoskeleton in health and in disease. Clin Kidney J. 5:498-501.

Matsumoto, N., K. Kita, S. Kojima, K. Yamamoto, T. Irimura, M. Miyagi, S. Tsunasawa, and S. Toyoshima. 1995a. Lymphocyte isoforms of mouse p50 LSP1, which are phosphorylated in mitogen-activated T cells, are formed through alternative splicing and phosphorylation. Journal of biochemistry. 118:237-243.

Matsumoto, N., S. Kojima, T. Osawa, and S. Toyoshima. 1995b. Protein kinase C phosphorylates p50 LSP1 and induces translocation of p50 LSP1 in T lymphocytes. Journal of biochemistry. 117:222-229.

Matsumoto, N., S. Toyoshima, and T. Osawa. 1993. Characterization of the $50 \mathrm{kDa}$ protein phosphorylated in concanavalin A-stimulated mouse T cells. Journal of biochemistry. 113:630-636.

Maxeiner, S., N. Shi, C. Schalla, G. Aydin, M. Hoss, S. Vogel, M. Zenke, and A.S. Sechi. 2015. Crucial role for the LSP1-myosin1e bimolecular complex in the regulation of Fcgamma receptor-driven phagocytosis. Molecular biology of the cell. 26:1652-1664.

May, R.C., M.E. Hall, H.N. Higgs, T.D. Pollard, T. Chakraborty, J. Wehland, L.M. Machesky, and A.S. Sechi. 1999. The Arp2/3 complex is essential for the actinbased motility of Listeria monocytogenes. Current biology: CB. 9:759--762.

Meijering, E., O. Dzyubachyk, and I. Smal. 2012. Methods for cell and particle tracking. Methods Enzymol. 504:183-200.

Miyoshi, E.K., P.L. Stewart, P.W. Kincade, M.B. Lee, A.A. Thompson, and R. Wall. 2001. Aberrant expression and localization of the cytoskeleton-binding pp52 (LSP1) protein in hairy cell leukemia. Leukemia research. 25:57-67.

Palker, T.J., A.M. Fong, R.M. Scearce, D.D. Patel, and B.F. Haynes. 1998. Developmental regulation of lymphocyte-specific protein 1 (LSP1) expression in thymus during human T-cell maturation. Hybridoma. 17:497-507. 
Petri, B., J. Kaur, E.M. Long, H. Li, S.A. Parsons, S. Butz, M. Phillipson, D. Vestweber, K.D. Patel, S.M. Robbins, and P. Kubes. 2011. Endothelial LSP1 is involved in endothelial dome formation, minimizing vascular permeability changes during neutrophil transmigration in vivo. Blood. 117:942-952.

Prasad, A., R. Kulkarni, S. Jiang, and J.E. Groopman. 2017. Cocaine Enhances DC to T-cell HIV-1 Transmission by Activating DC-SIGN/LARG/LSP1 Complex and Facilitating Infectious Synapse Formation. Sci Rep. 7:40648.

Prasad, A., P.M. Kuzontkoski, A. Shrivastava, W. Zhu, D.Y. Li, and J.E. Groopman. 2012. Slit2N/Robo1 inhibit HIV-gp120-induced migration and podosome formation in immature dendritic cells by sequestering LSP1 and WASp. PloS one. 7:e48854.

Pust, S., H. Morrison, J. Wehland, A.S. Sechi, and P. Herrlich. 2005. Listeria monocytogenes exploits ERM protein functions to efficiently spread from cell to cell. The EMBO journal. 24:1287--1300.

Ramaekers, F.C., and F.T. Bosman. 2004. The cytoskeleton and disease. J Pathol. 204:351-354.

Rapsomaniki, M.A., P. Kotsantis, I.E. Symeonidou, N.N. Giakoumakis, S. Taraviras, and Z. Lygerou. 2012. easyFRAP: an interactive, easy-to-use tool for qualitative and quantitative analysis of FRAP data. Bioinformatics. 28:1800-1801.

Schulze, N., M. Graessl, A. Blancke Soares, M. Geyer, L. Dehmelt, and P. Nalbant. 2014. FHOD1 regulates stress fiber organization by controlling the dynamics of transverse arcs and dorsal fibers. Journal of cell science. 127:1379-1393.

Sechi, A., J.M.G. Freitas, P. Wunnemann, A. Topel, R.T. Paschoalin, S. Ullmann, R. Schroder, G. Aydin, S. Rutten, A. Boker, M. Zenke, and A. Pich. 2016. SurfaceGrafted Nanogel Arrays Direct Cell Adhesion and Motility. Adv Mater Interfaces. 3.

Sechi, A.S., and J. Wehland. 2004. ENA/VASP proteins: multifunctional regulators of actin cytoskeleton dynamics. Front Biosci. 9:1294--1310.

Sirotkin, V., C.C. Beltzner, J.B. Marchand, and T.D. Pollard. 2005. Interactions of WASp, myosin-I, and verprolin with Arp2/3 complex during actin patch assembly in fission yeast. The Journal of cell biology. 170:637-648.

Tanimura, S., J. Hashizume, N. Arichika, K. Watanabe, K. Ohyama, K. Takeda, and M. Kohno. 2016. ERK signaling promotes cell motility by inducing the localization of myosin 1E to lamellipodial tips. The Journal of cell biology. 214:475-489.

Wang, C., H. Hayashi, R. Harrison, B. Chiu, J.R. Chan, H.L. Ostergaard, R.D. Inman, J. Jongstra, M.I. Cybulsky, and J. Jongstra-Bilen. 2002. Modulation of Mac-1 (CD11b/CD18)-mediated adhesion by the leukocyte-specific protein 1 is key to its role in neutrophil polarization and chemotaxis. Journal of immunology. 169:415-423.

Way, M. 1998. Interaction of vaccinia virus with the actin cytoskeleton. Folia Microbiol (Praha). 43:305-310. 
Wehland, J., M.C. Willingham, and I.V. Sandoval. 1983. A rat monoclonal antibody reacting specifically with the tyrosylated form of alpha-tubulin. I. Biochemical characterization, effects on microtubule polymerization in vitro, and microtubule polymerization and organization in vivo. The Journal of cell biology. 97:1467-1475.

Wong, M.J., I.A. Malapitan, B.A. Sikorski, and J. Jongstra. 2003. A cell-free binding assay maps the LSP1 cytoskeletal binding site to the $\mathrm{COOH}$-terminal 30 amino acids. Biochimica et biophysica acta. 1642:17-24.

Wu, Y., L. Zhan, Y. Ai, M. Hannigan, M. Gaestel, C.K. Huang, and J.A. Madri. 2007. MAPKAPK2-mediated LSP1 phosphorylation and FMLP-induced neutrophil polarization. Biochemical and biophysical research communications. 358:170175.

Würflinger, T., I. Gamper, T. Aach, and A.S. Sechi. 2011. Automated segmentation and tracking for large-scale analysis of focal adhesion dynamics. Journal of Microscopy. 241:37--53.

Zamir, E., and B. Geiger. 2001. Molecular complexity and dynamics of cell-matrix adhesions. Journal of cell science. 114:3583-3590.

Zhang, Q., Y. Li, and T.H. Howard. 2000. Human lymphocyte-specific protein 1, the protein overexpressed in neutrophil actin dysfunction with 47-kDa and $89-\mathrm{kDa}$ protein abnormalities (NAD 47/89), has multiple F-actin binding domains. Journal of immunology. 165:2052-2058.

Zhang, Q., Y. Li, and T.H. Howard. 2001. Hair-forming activity of human lymphocyte specific protein 1 requires cooperation between its caldesmon-like domains and the villin headpiece-like domains. Cell motility and the cytoskeleton. 49:179-188. 


\section{Figure legends}

Figure 1. LSP1 is essential for macrophage cell migration. (A, B) Control and LSP1deficient $\mathbf{J 7 7 4}$ were cultured on glass coverslips for 24 hours before being imaged by phase contrast microscopy. Control J774 cells characteristically developed large lamellipodia and travelled large distances (arrows in A). By contrast, LSP1-deficient J774 cells (LSP1-KD) did not form lamellipodia and displayed reduced migration (arrows in B). Lower panel in B (GFP) shows the expression of LSP1-specific shRNA. Numbers indicate the elapsed time in hours, minutes and seconds. (C, D) Representative migration tracks of control (C) and LSP1-deficient (D) J774 cells. Scale bars (A-D): $100 \mu \mathrm{m}$. (E) Quantification of the average speed of control and LSP1deficient $\mathrm{J774}$ cells. Box-and-whiskers plots. The line in the middle of the box indicates the median, the top of the box indicates the $75^{\text {th }}$ quartile, and the bottom of the box indicates the $25^{\text {th }}$ quartile. Whiskers represent the $10^{\text {th }}$ (lower) and $90^{\text {th }}$ (upper) percentiles. ${ }^{\star \star \star \star} p<0.0001$

Figure 2. LSP1 is essential for normal development of microfilaments, microtubules and focal adhesions. (A, B) Microtubule and EB1 cytoplasmic distribution in control and LSP1-deficient (LSP1-KD) J774 cells. Following fixation, microtubules and EB1 were visualised using anti-tubulin and anti-EB1 antibodies, respectively. Samples were analysed by TIRF microscopy. In control cells (A), microtubules were welldeveloped typically originating from a perinuclear area and projecting towards cell periphery (left panel, arrows in inset). EB1 characteristically localised at the peripheral tips of microtubules (central and right panels, arrows in insets). LSP1-deficient cells acquired a rounded shape and were characterised by shorter microtubules ( $B$, left panel, arrow in inset). EB1 localisation was not grossly changed. Right panels show merged microtubule (shown in green) and EB1 (shown in red) images. Boxes indicate the areas enlarged in the insets. Scale bar: $10 \mu \mathrm{m}$. (C, D) Actin cytoskeleton and 
focal adhesion distribution in control and LSP1-deficient (LSP1-KD) J774 cells. The actin cytoskeleton was visualised using fluorescent phalloidin, whereas focal adhesions were detected using anti-vinculin antibodies. Samples were analysed by TIRF microscopy. Control cells formed one or more large actin-rich lamellipodia (C, left panel, green arrowheads). Conversely, LSP1-deficient cells formed only small lamellipodia ( $D$, left panel, red arrowhead). Focal adhesions were also altered in LSP1-deficient cells, which formed fewer and smaller focal adhesions than control cells (arrows in central and right panels). Right panels show merged actin (shown in green) and vinculin (shown in red) images. Scale bar: $10 \mu \mathrm{m}$.

Figure 3. LSP1 is essential for the regulation of focal adhesion dynamics. (A, B) Representative time-lapse images showing focal adhesion dynamics in control (A) and LSP1-deficient (B; LSP1-KD) J774 cells. Focal adhesions were visualised using RFPzyxin and images were acquired by TIRF microscopy. Note the faster turnover of focal adhesions in control cells (arrows in A) compared to focal adhesions in LSP1-deficient cells (arrows in B). Numbers indicate the elapsed time in minutes and seconds. Scale bar: $10 \mu \mathrm{m}$. (C-H) Quantification of focal adhesion parameters. In the box-andwhiskers plots the line in the middle of the box indicates the median, the top of the box indicates the $75^{\text {th }}$ quartile, and the bottom of the box indicates the $25^{\text {th }}$ quartile. Whiskers represent the $10^{\text {th }}$ (lower) and $90^{\text {th }}$ (upper) percentiles. ns: non-significant.

Figure 4. LSP1-myosin1e interaction is essential for $\mathrm{J774}$ cell migration. (A, B) J774 cells expressing the LSP1- $\triangle$ SBS (A) or full-length LSP1 (B; LSP1_WT rescue) were cultured on glass coverslips for 24 hours before being imaged by phase contrast microscopy. J774 cells in which the expression of full-length LSP1 was restored (rescued; arrows in B) moved at faster speed compared to cells expressing the LSP1 mutant LSP1- $\triangle$ SBS, which is unable to interact with myosin 1e (arrows in A). Numbers indicate the elapsed time in hours, minutes and seconds. (C, D) Representative 
migration tracks of rescue (C) and LSP1- $\triangle$ SBS (D) J774 cells. Scale bars (A-D): 100 $\mu \mathrm{m}$. (E) Quantification of the average speed of LSP1- $\triangle$ SBS and rescue J774 cells. For easier comparison, the average speeds of control and LSP1-deficient cells (data from Fig. 1) are also plotted. Box-and-whiskers plots. The line in the middle of the box indicates the median, the top of the box indicates the $75^{\text {th }}$ quartile, and the bottom of the box indicates the $25^{\text {th }}$ quartile. Whiskers represent the $10^{\text {th }}$ (lower) and $90^{\text {th }}$ (upper) percentiles.

Figure 5. LSP1-myosin1e complex is essential for lamellipodia formation and dynamics. (A-C) Time-lapse images showing lamellipodia morphology and dynamics in control (A), LSP1-deficient (B; LSP1-KD) and J774 cells expressing the LSP1 deletion mutant LSP1- $\triangle$ SBS (C). Polarised control cells typically formed one large and very dynamic lamellipodium in the direction of movement (arrows in A). By contrast, LSP1deficient cells or cells expressing an LSP1 mutant unable to interact with myosin 1e acquired a rounded morphology and formed very small lamellipodia around their periphery (arrows in B, C). Numbers indicate the elapsed time in minutes and seconds. Scale bar: $10 \mu \mathrm{m}$. (D-F) Box-and-whiskers plots showing the quantification of width, spreading velocity and frequency of lamellipodia formation. The line in the middle of the box indicates the median, the top of the box indicates the $75^{\text {th }}$ quartile, and the bottom of the box indicates the $25^{\text {th }}$ quartile. Whiskers represent the $10^{\text {th }}$ (lower) and $90^{\text {th }}$ (upper) percentiles.

Figure 6. LSP1-myosin1e interaction regulates the dynamics and kinetics of LSP1. (AC) TIRF imaging showing the dynamics of LSP1 in control J774 cells (A), cells expressing the LSP1 mutant unable to interact with myosin 1e (LSP1- $\Delta$ SBS; B) or fulllength LSP1 (rescue; C). Note the intense dynamics of LSP1 in control (A) and rescue cells (C) and the localisation of LSP1 to filamentous-like structures (red arrows in A) and focal adhesions-like structures (green arrows in A, orange arrows in C). Stars 
indicate lamellipodia. In cells expressing LSP1- $\triangle$ SBS, LSP1 robustly localises around the nucleus (white arrow in B) and at very small lamellipodia (yellow arrows in B). Numbers indicate the elapsed time in minutes and seconds. Scale bar: $10 \mu \mathrm{m}$. (D, E) Quantification of the fluorescence recovery (D) and mobile fraction (E) of LSP1. Error bars show s.e.m. In the box-and-whiskers plots, the line in the middle of the box indicates the median, the top of the box indicates the $75^{\text {th }}$ quartile, and the bottom of the box indicates the $25^{\text {th }}$ quartile. Whiskers represent the $10^{\text {th }}$ (lower) and $90^{\text {th }}$ (upper) percentiles.

Figure 7. LSP1-myosin1e interaction is essential for efficient focal adhesion dynamics in $\mathrm{J774}$ cells. (A, B) J774 cells expressing full-length LSP1 (A; rescue) or the LSP1 mutant unable to interact with myosin 1e (B; LSP1- $\triangle$ SBS) were transfected with RFPzyxin to visualise focal adhesions and images were acquired by TIRF microscopy. Note the faster turnover of focal adhesions in cells expressing full-length LSP1 (arrows in A) compared to focal adhesions in cells expressing the LSP1 mutant unable to interact with myosin 1e (arrows in B). Numbers indicate the elapsed time in minutes and seconds. Scale bar: $10 \mu \mathrm{m}$. (C-H) Quantification of focal adhesion parameters. In the box-and-whiskers plots the line in the middle of the box indicates the median, the top of the box indicates the $75^{\text {th }}$ quartile, and the bottom of the box indicates the $25^{\text {th }}$ quartile. Whiskers represent the $10^{\text {th }}$ (lower) and $90^{\text {th }}$ (upper) percentiles. ns: nonsignificant.

Figure 8. LSP1-myosin1e interaction regulates the amount of actin-associated vinculin. (A) Soluble and cytoskeletal fractions from control (WT), LSP1-deficient (LSP1-KD) and J774 cells expressing the LSP1- $\triangle$ SBS mutant (unable to interact with myosin1e) resolved by SDS-PAGE and probed with antibodies against vinculin, LSP1 and actin, which served as loading control. Numbers on the left side indicate MW markers in kDa. Note the reduced amount of vinculin recovered in the cytoskeleton 
fraction of LSP1-deficient cells and cells expressing the deletion mutant LSP1- $\triangle$ SBS. (B) Quantification of vinculin/actin ratio in cytoskeleton fraction. Vinculin/actin ratio for control cells (WT) was set to 1 . Columns show mean \pm s.e.m $(n=3)$.

Figure 9. LSP1-myosin1e interaction is essential for efficient kinetics of zyxin at focal adhesions. (A-D) Control J774 cells (A), LSP1-deficient cells (LSP1-KD; B), cells expressing the LSP1 mutant unable to interact with myosin 1e (LSP1- $\Delta$ SBS; C) or fulllength LSP1 (rescue; D) were stably transfected with RFP-zyxin to visualise focal adhesions. RFP-zyxin kinetics was determined by fluorescence recovery after photobleaching. Arrows in A-D point to bleached focal adhesions. Note the slower recovery of the fluorescence signal at bleached focal adhesions in LSP1-deficient cells (B) and in cells expressing the LSP1 mutant unable to interact with myosin1e (C). (E, F) Quantification of the fluorescence recovery (E) and mobile fraction (F) of RFP-zyxin. Error bars show s.e.m. In the box-and-whiskers plots, the line in the middle of the box indicates the median, the top of the box indicates the $75^{\text {th }}$ quartile, and the bottom of the box indicates the $25^{\text {th }}$ quartile. Whiskers represent the $10^{\text {th }}$ (lower) and $90^{\text {th }}$ (upper) percentiles.

Figure 10. Schematic model for the role of LSP1-myosin1e complex during cell migration and adhesion. The LSP1-myosin1e binary complex could regulate cell adhesion and migration via three different, but not necessarily functionally independent, signalling pathways. The LSP1-myosin1e complex could regulate actin cytoskeleton contraction through calmodulin and myosin light chain kinase, which modulate myosin II activity. Actin filament reorganisation and FA maturation could be controlled via FHOD1. Finally, the LSP1-myosin1e could also impinge on actin filament assembly upon the regulation of WASP family proteins and the Arp $2 / 3$ complex. The functional integration of these three signalling pathways will efficiently 
bioRxiv preprint doi: https://doi.org/10.1101/2020.02.26.963991; this version posted February 27, 2020. The copyright holder for this

preprint (which was not certified by peer review) is the author/funder. All rights reserved. No reuse allowed without permission.

regulate actin cytoskeleton remodelling, FA and lamellipodia dynamics leading to efficient cell migration. 

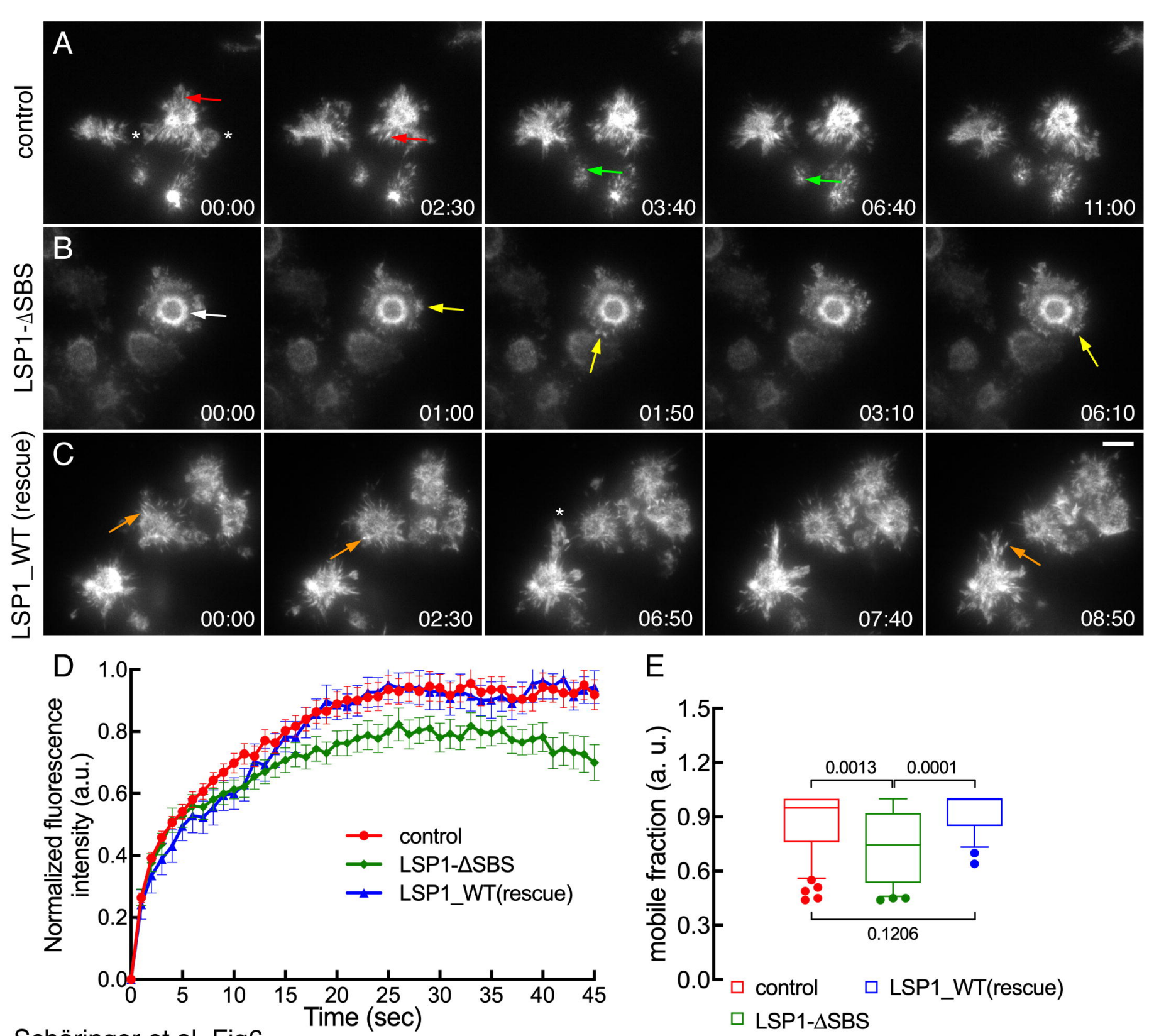

E

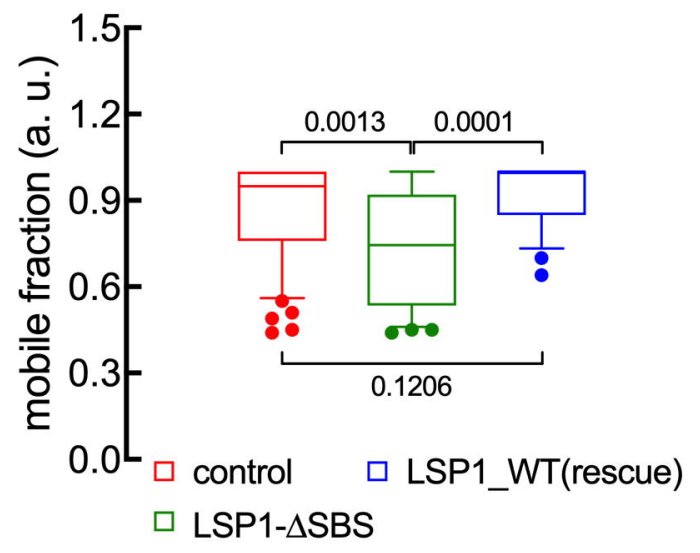

Schäringer et al. Fig6 
0
$\frac{1}{0}$
0
0
5
5
5
0

A
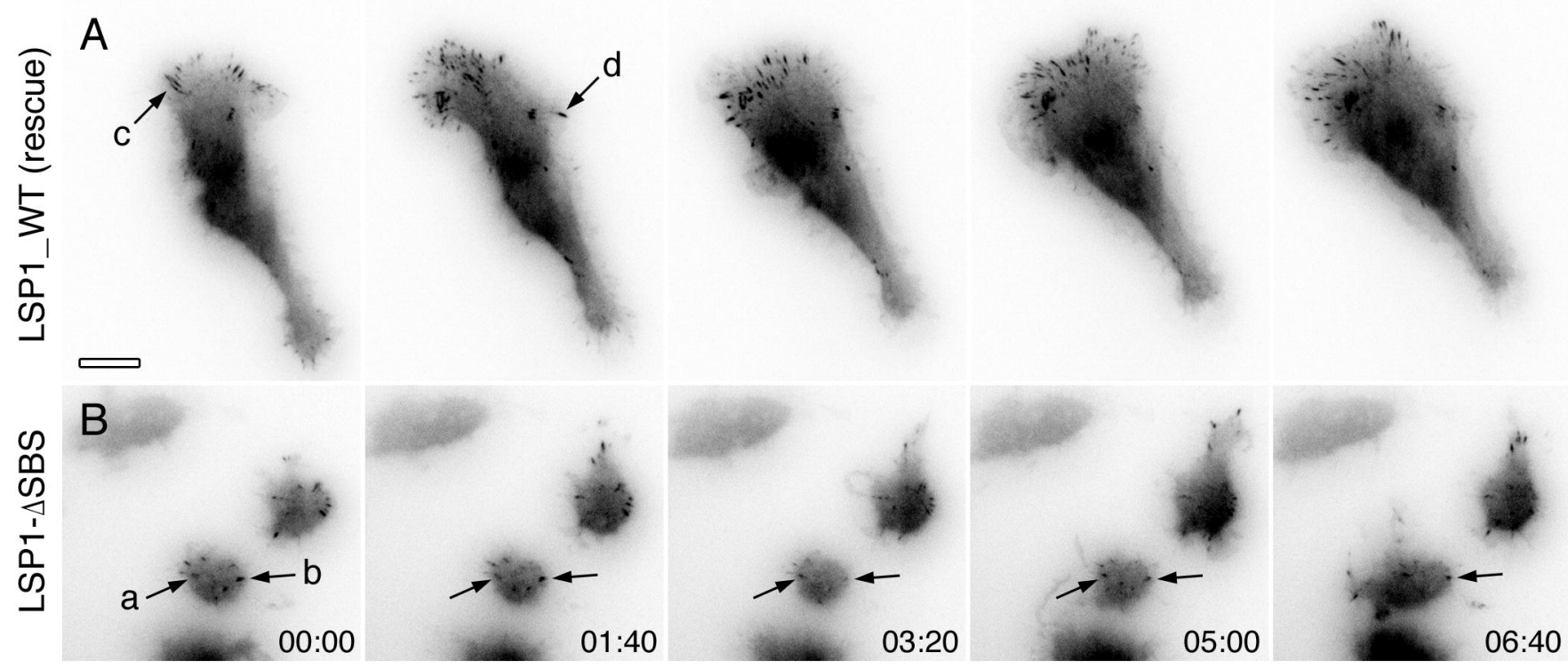

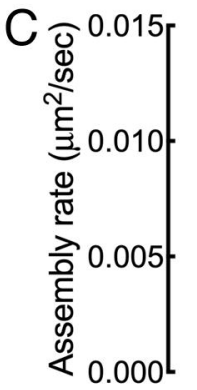

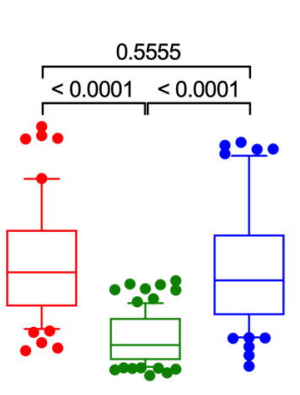

D ত্ষ 0.015
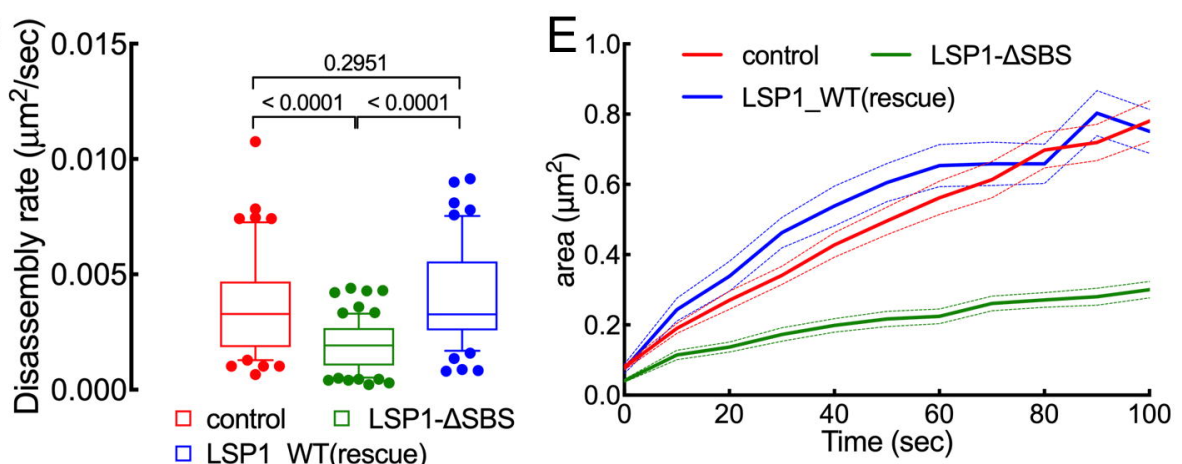

$\mathrm{F}$
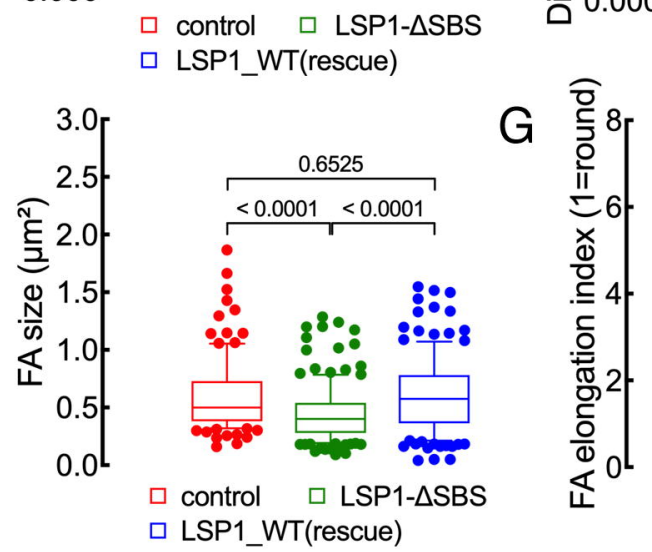

$\square$ control $\square$ LSP1- $\triangle$ SBS

口 LSP1_WT(rescue)

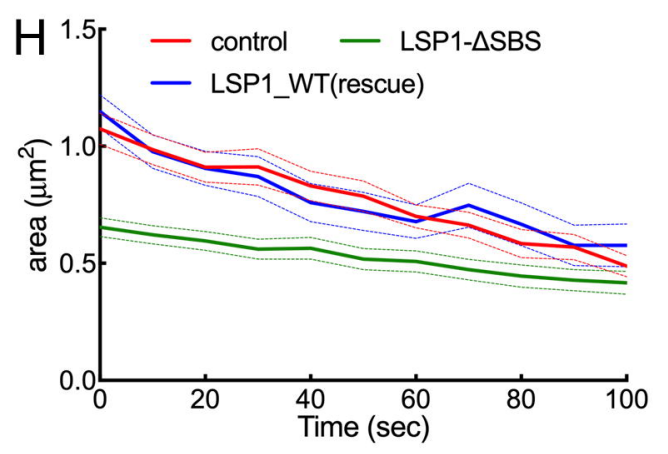

Schäringer et al. Fig7 


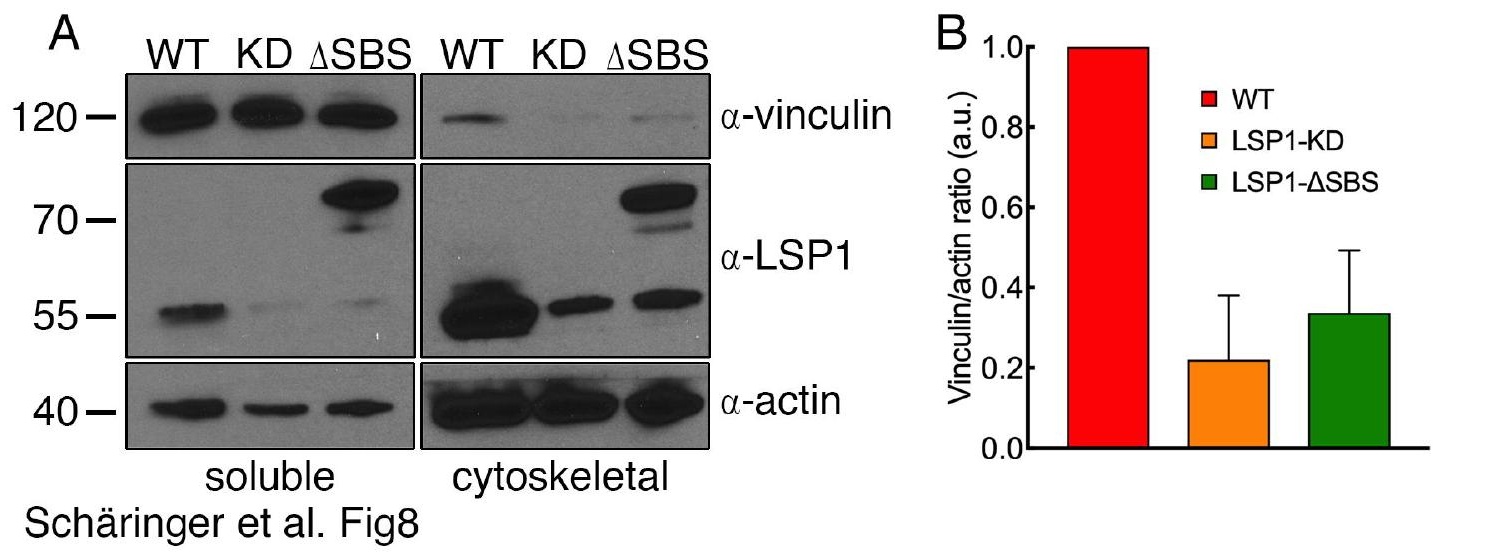


A

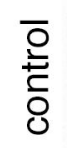

$\frac{1}{4}$

00:00

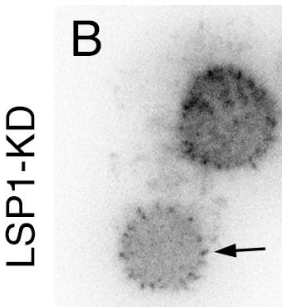

00:00

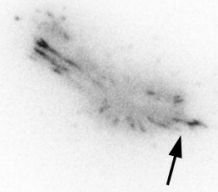

00:03

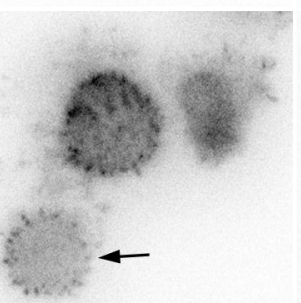

00:03

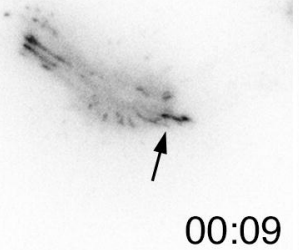

00:09

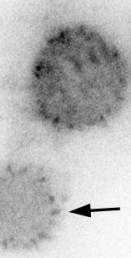

00:40

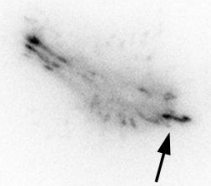

00:19

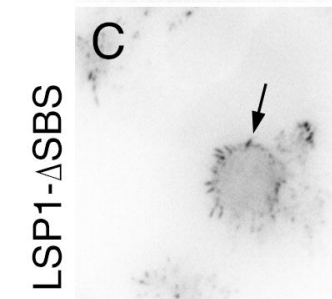

00:00
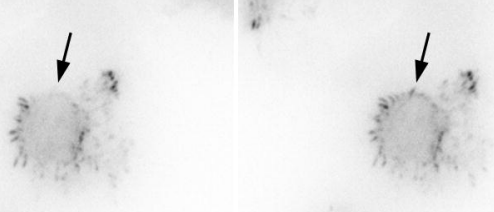

00:01

00:30

02:00

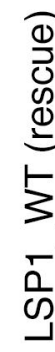

ब1
0
0
0
0
3
0
0
0
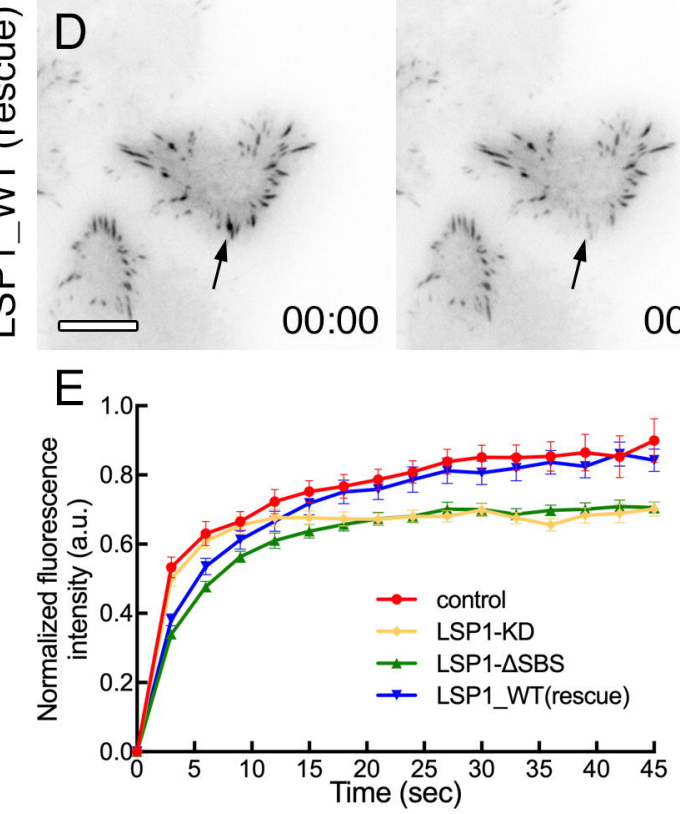

00:01
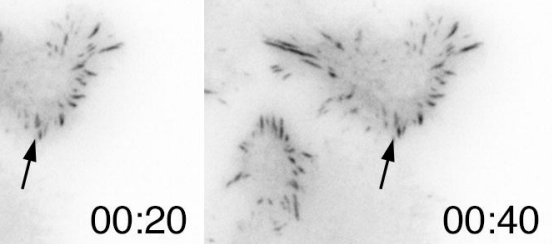

Schäringer et al. Fig9

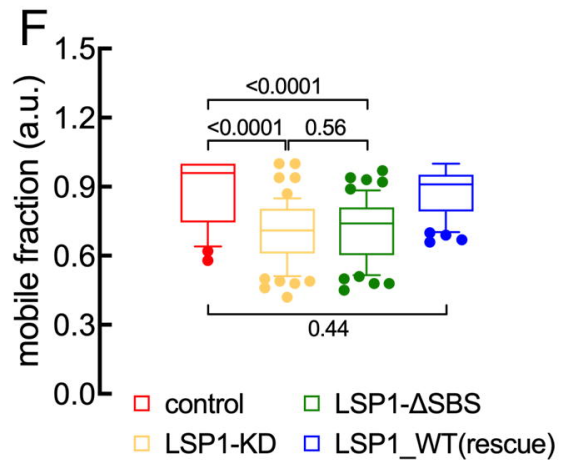


calmodulin, MLCK

$$
\downarrow
$$

actin cytoskeleton contraction

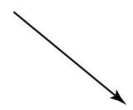

actin cytoskeleton remodelling, FA and lamellipodia dynamics

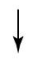

WIP, N-WASP, WASP

FHOD1

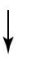

actin filament reorganisation, FA maturation actin filament

- assembly

Arp2/3 complex

$\downarrow$

Schäringer et al. Fig10 